\title{
Globally Optimal Design Optimization of Cooling Water System
}

Ana L. L. Levy, ${ }^{+}$Jaime N. M. Souza, ${ }^{\ddagger}$ Miguel J. Bagajewicz, ${ }^{++}$André L. H. Costa ${ }^{*+}$

${ }^{+}$Institute of Chemistry, Rio de Janeiro State University (UERJ), Rua São Francisco Xavier, 524, Maracanã, Rio de Janeiro, RJ, CEP 20550-900, Brazil

${ }^{\ddagger}$ LEPABE, Department of Chemical Engineering, Faculty of Engineering, University of Porto, Rua Dr. Roberto Frias, s/n, 4200-465 Porto, Portugal

${ }^{++}$School of Chemical, Biological and Materials Engineering, University of Oklahoma, Norman Oklahoma 73019 


\section{Supporting Information}

This document contains the full description of the results of Example 2.

Table S1. Example 2: Heat exchanger design results (he1)

\begin{tabular}{cccc}
\hline & Simultaneous design & Two-step design A & Two-step design B \\
\hline Area $\left(\mathrm{m}^{2}\right)$ & 123.5 & 113.9 & 123.5 \\
Tube diameter $(\mathrm{m})$ & 0.019 & 0.019 & 0.019 \\
Tube length $(\mathrm{m})$ & 3.049 & 2.439 & 3.049 \\
Number of baffles & 15 & 14 & 18 \\
Number of tube passes & 2 & 4 & 2 \\
Tube pitch ratio & 1.25 & 1.25 & 1.25 \\
Shell diameter (m) & 0.686 & 0.737 & 0.686 \\
Tube layout & 2 & 2 & 2 \\
Total number of tubes & 677 & 781 & 677 \\
Baffle spacing (m) & 0.191 & 0.163 & 0.160 \\
\hline
\end{tabular}

Table S2. Example 2: Heat exchanger design results (he2)

\begin{tabular}{cccc}
\hline & Simultaneous design & Two-step design A & Two-step design B \\
\hline Area $\left(\mathrm{m}^{2}\right)$ & 246.6 & 227.8 & 211.5 \\
Tube diameter $(\mathrm{m})$ & 0.019 & 0.019 & 0.019 \\
Tube length $(\mathrm{m})$ & 6.098 & 4.877 & 6.098 \\
Number of baffles & 8 & 8 & 9 \\
Number of tube passes & 6 & 6 & 6 \\
Tube pitch ratio & 1.25 & 1.25 & 1.25 \\
Shell diameter (m) & 0.737 & 0.737 & 0.635 \\
Tube layout & 1 & 2 & 2 \\
Total number of tubes & 676 & 781 & 580 \\
Baffle spacing $(\mathrm{m})$ & 0.678 & 0.542 & 0.610 \\
\hline
\end{tabular}


Table S3. Example 2: Heat exchanger design results (he3)

\begin{tabular}{cccc}
\hline & Simultaneous design & Two-step design A & Two-step design B \\
\hline Area $\left(\mathrm{m}^{2}\right)$ & 197.5 & 207.3 & 197.5 \\
Tube diameter $(\mathrm{m})$ & 0.019 & 0.019 & 0.019 \\
Tube length (m) & 4.877 & 3.049 & 4.877 \\
Number of baffles & 17 & 9 & 17 \\
Number of tube passes & 2 & 4 & 2 \\
Tube pitch ratio & 1.25 & 1.25 & 1.25 \\
Shell diameter (m) & 0.686 & 0.889 & 0.686 \\
Tube layout & 2 & 2 & 2 \\
Total number of tubes & 677 & 1137 & 677 \\
Baffle spacing (m) & 0.271 & 0.305 & 0.271 \\
\hline
\end{tabular}

Table S4. Example 2: Heat exchanger design results (he4)

\begin{tabular}{cccc}
\hline & Simultaneous design & Two-step design A & Two-step design B \\
\hline Area $\left(\mathrm{m}^{2}\right)$ & 75.3 & 61.1 & 75.3 \\
Tube diameter $(\mathrm{m})$ & 0.019 & 0.019 & 0.019 \\
Tube length $(\mathrm{m})$ & 3.659 & 2.439 & 3.659 \\
Number of baffles & 15 & 17 & 17 \\
Number of tube passes & 2 & 4 & 2 \\
Tube pitch ratio & 1.25 & 1.25 & 1.25 \\
Shell diameter (m) & 0.489 & 0.540 & 0.489 \\
Tube layout & 2 & 2 & 2 \\
Total number of tubes & 344 & 419 & 677 \\
Baffle spacing (m) & 0.229 & 0.136 & 0.261 \\
\hline
\end{tabular}


Table S5. Example 2: Diameters and head loss of the pipes

\begin{tabular}{ccccccc}
\hline Pipe & \multicolumn{2}{c}{ Simultaneous design } & \multicolumn{2}{c}{ Two-step design A } & \multicolumn{2}{c}{ Two-step design B } \\
\cline { 2 - 7 } & $\begin{array}{c}\text { Diameter } \\
\text { (in) }\end{array}$ & $\begin{array}{c}\text { Head loss } \\
(\mathrm{m})\end{array}$ & $\begin{array}{c}\text { Diameter } \\
\text { (in) }\end{array}$ & $\begin{array}{c}\text { Head loss } \\
(\mathrm{m})\end{array}$ & $\begin{array}{c}\text { Diameter } \\
\text { (in) }\end{array}$ & $\begin{array}{c}\text { Head loss } \\
\text { (m) }\end{array}$ \\
pi1 & 20 & 0.568 & 16 & 1.710 & 16 & 1.710 \\
pi2 & 8 & 0.507 & 10 & 0.167 & 8 & 0.507 \\
pi3 & 16 & 0.177 & 16 & 0.177 & 14 & 0.340 \\
pi4 & 10 & 0.123 & 8 & 0.372 & 10 & 0.123 \\
pi5 & 8 & 0.372 & 8 & 0.372 & 10 & 0.123 \\
pi6 & 8 & 0.507 & 8 & 0.507 & 8 & 0.507 \\
pi7 & 14 & 0.202 & 12 & 0.319 & 10 & 0.750 \\
pi8 & 8 & 0.194 & 8 & 0.194 & 8 & 0.194 \\
pi9 & 12 & 0.050 & 8 & 0.359 & 8 & 0.359 \\
pi10 & 12 & 0.050 & 8 & 0.359 & 8 & 0.359 \\
pi11 & 8 & 0.108 & 8 & 0.108 & 6 & 0.410 \\
pi12 & 8 & 0.108 & 8 & 0.108 & 8 & 0.108 \\
pi13 & 8 & 0.194 & 8 & 0.194 & 6 & 0.738 \\
pi14 & 12 & 0.319 & 12 & 0.319 & 10 & 0.750 \\
pi15 & 18 & 0.100 & 16 & 0.177 & 16 & 0.177 \\
pi16 & 18 & 0.982 & 18 & 0.982 & 16 & 1.745 \\
pi17 & 20 & 0.012 & 18 & 0.020 & 16 & 0.035 \\
\hline
\end{tabular}

Table S6. Example 2: Pump head

\begin{tabular}{cc}
\hline Design & Pump head (m) \\
\hline Simultaneous design & 6 \\
Two-step design (a) & 10 \\
Two-step design (b) & 10 \\
\hline
\end{tabular}

Table S7. Example 2: Head loss in the valves

\begin{tabular}{ccccc}
\hline Head loss (m) & he1 & he2 & he3 & he4 \\
\hline Simultaneous design & 0.450 & 0.017 & 0.024 & 0.027 \\
Two-step design (a) & 0.599 & 0.588 & 0.726 & 0.008 \\
Two-step design (b) & 2.522 & 0.102 & 0.259 & 0.033 \\
\hline
\end{tabular}


Table S8. Example 2: Annualized costs

\begin{tabular}{cccc}
\hline Cost $(\$ /$ year $)$ & Simultaneous design & Two-step design A & Two-step design B \\
\hline Pump cost & 1455.21 & 1752.81 & 1752.81 \\
Heat exchanger cost & 48350.33 & 46232.95 & 46253.56 \\
Pipe cost & 15606.02 & 13591.52 & 12354.19 \\
Operational cost & 20739.07 & 34565.12 & 34565.12 \\
Total cost & 86150.64 & 96142.40 & 94925.69 \\
\hline
\end{tabular}

